\title{
Learning Drives Differential Clustering of Axodendritic Contacts in the Barn Owl Auditory System
}

\author{
Thomas J. McBride, Adrian Rodriguez-Contreras, Angela Trinh, Robert Bailey, and William M. DeBello \\ Center for Neuroscience, Department of Neurobiology, Physiology and Behavior, University of California, Davis, Davis, California 95618
}

Computational models predict that experience-driven clustering of coactive synapses is a mechanism for information storage. This prediction has remained untested, because it is difficult to approach through time-lapse analysis. Here, we exploit a unique feature of the barn owl auditory localization pathway that permits retrospective analysis of prelearned and postlearned circuitry: owls reared wearing prismatic spectacles develop an adaptive microcircuit that coexists with the native one but can be analyzed independently based on topographic location. To visualize the clustering of axodendritic contacts (potential synapses) within these zones, coactive axons were labeled by focal injection of fluorescent tracer and their target dendrites labeled with an antibody directed against CaMKII (calcium/ calmodulin-dependent protein kinase type II, $\alpha$ subunit). Using high-resolution confocal imaging, we measured the distance from each contact to its nearest neighbor on the same branch of dendrite. We found that the distribution of intercontact distances for the adaptive zone was shifted dramatically toward smaller values compared with distributions for either the maladaptive zone of the same animals or the adaptive zone of normal juveniles, which indicates that a dynamic clustering of contacts had occurred. Moreover, clustering in the normal zone was greater in normal juveniles than in prism-adapted owls, indicative of declustering. These data demonstrate that clustering is bidirectionally adjustable and tuned by behaviorally relevant experience. The microanatomical configurations in all zones of both experimental groups matched the functional circuit strengths that were assessed by in vivo electrophysiological mapping. Thus, the observed changes in clustering are appropriately positioned to contribute to the adaptive strengthening and weakening of auditorydriven responses.

Key words: remodeling; synapse clustering; inferior colliculus; learning; microcircuit; plasticity

\section{Introduction}

Learning depends on the long-term modification of brain circuits. Experience-driven regulation of synaptic strength likely plays an important role in this modification, but the capacity of weight changes to store information is limited in theory compared with the rewiring of connections (Poirazi and Mel, 2001; Chklovskii et al., 2004). Recent vital imaging studies have established that synapse formation and elimination can occur in the brains of several species (Hua and Smith, 2004; Nagerl et al., 2004; De Paola et al., 2006; Holtmaat et al., 2006; Lee et al., 2006; Stettler et al., 2006). Whether learning harnesses these structural changes to alter the relevant microwiring diagrams in functionally significant ways is unknown.

One structural motif that could contribute to optimized processing is the physical clustering of coactive synapses onto individual dendritic branches (Poirazi and Mel, 2001). Clustered synapses are expected to interact via supralinear summation, according to both biophysical models (Poirazi et al., 2003) and electrophysiological data gathered from neocortical pyramidal

Received Jan. 10, 2008; accepted April 12, 2008.

This work was supported by National Institute on Deafness and Other Communication Disorders-National Institutes of Health Grant R01 DC05640 (W.M.D.).

Correspondence should be addressed to William M. DeBello, Center for Neuroscience, University of California, Davis, 1544 Newton Court, Davis, CA 95618. E-mail:wmdebello@ucdavis.edu.

DOI:10.1523/JNEUROSCI.1352-08.2008

Copyright $\odot 2008$ Society for Neuroscience $\quad$ 0270-6474/08/286960-14\$15.00/0 cells (Polsky et al., 2004; Gasparini and Magee, 2006). The implications of these biophysical results for learning are illustrated by a thought experiment proposed by Poirazi and Mel. In it, a postsynaptic neuron is "taught" to distinguish among input patterns through the gradual clustering of coactive synapses as dendritic neighbors (Poirazi and Mel, 2001). Here, we provide the first test of this hypothesis by analyzing microstructural changes within the well characterized circuits of the barn owl auditory localization pathway.

Barn owls, like humans, localize sounds through the analysis of binaural cues (Konishi, 2003). This information is integrated by individual neurons located in the external nucleus of the inferior colliculus (ICX) (Knudsen, 1983). Space-specific neurons in ICX receive monosynaptic input from axons originating in the lateral shell of the central nucleus of the inferior colliculus (IC$\mathrm{Cls}$ ), and this connection is a major site of plasticity (Brainard and Knudsen, 1993). Specifically, when owls are exposed to chronic optical displacement of the visual field ("prism-rearing") (Knudsen and Knudsen, 1985), the topographic projection from ICCls and ICX is selectively sculpted (DeBello et al., 2001). This anatomical change is physiologically and behaviorally adaptive (Knudsen, 2002).

The ICCls-ICX microcircuit provides a testing ground for the experiment proposed by Poirazi and Mel. The ICCls axonal arbor, readily labeled by focal injection of tracer, represents a set of coactive synapses that are distributed anatomically along a con- 
A

Normal juvenile

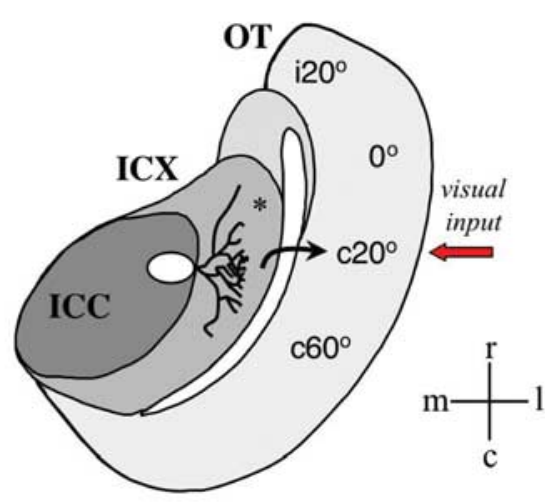

B
C

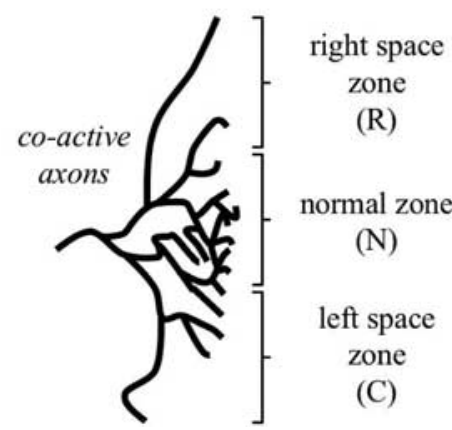

Prism-adapted

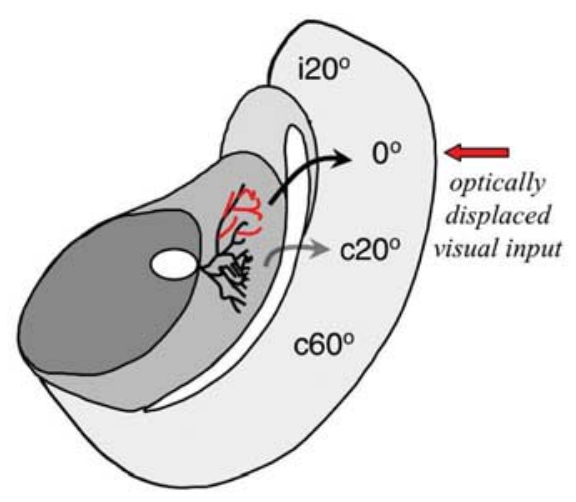

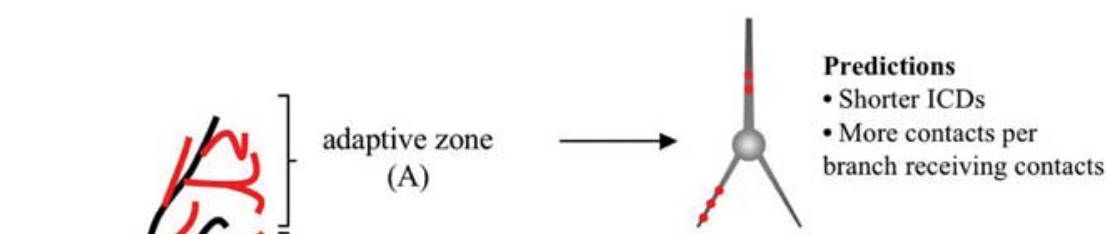

normal zone

(N)

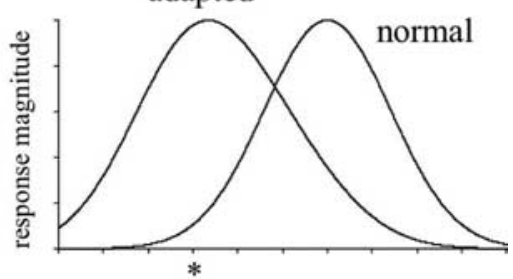

neuron location in ICX $(\mathrm{mm})$ $-1$

$-0.5$ 0.5

neuron's best auditory spatial tuning $\left(^{\circ}\right)$ $\begin{array}{llll}\text { i20 } & 0 & \text { c20 } & \text { c60 }\end{array}$

Figure 1. Hypothesis for microanatomical remodeling during prism-induced learning. $\boldsymbol{A}$, Schematic diagram of horizontal section through the R midbrain of a normal juvenile and prism-adapted owl. Stylized black (normal juvenile) or black and red (prism-adapted) lines depict the topographic axonal arbor representing contralateral $20^{\circ}$, projecting from the lateral shell of the ICC to the ICX. See C for additional explanation. Major postsynaptic targets of these axons in ICX are CaMKII + space-specific neurons (not depicted). The asterisk indicates the location of one space-specific neuron whose response properties are shown in $\boldsymbol{B}$. The curved arrows indicate the projection of CaMKII+ neurons to the 0 T where the auditory space map aligns with a visual space map. In both ICX and $0 \mathrm{~T}$, the maps of space are organized systematically with ipsilateral space (i.e., i $\left.20^{\circ}\right)$ represented in the rostral pole and contralateral space (i.e., $60^{\circ}$ ) toward the caudal pole. The red arrows indicate the location of visual input arising from a stimulus located $20^{\circ}$ to the owl's left, before (normal juvenile) and after (prism-adapted) prism mounting. $\boldsymbol{B}$, Distribution of auditory tunings of space-specific neurons in normal juveniles and prism-adapted owls. The neuron indicated by the asterisk (located in the adaptive zone) exhibits weak responses to stimulation of the $c 20^{\circ}$ axons in a normal juvenile, but strong responses to the same stimulation in prism-adapted owls. Conversely, neurons located 300-800 $\mu \mathrm{m}$ more caudal (in the normal zone) exhibit strong responses in normal juveniles but weaker responses in prism-adapted owls. C, Magnified view of axonal arbors illustrating the zones for microanatomical analysis. The black stylized lines (normal juvenile and prism-adapted) depict native axons. The red stylized lines (prism-adapted) depict the prism-induced adaptive axons and synapses. Note the persistence of normally targeted and maladaptive axons in prism-adapted owls. Our hypothesis predicts greater contact clustering in the adaptive zone than in the maladaptive zone.

tinuum of adaptive value: the rostral zone of the arbor represents learned, adaptive synapses, the central zone normal, formally adaptive synapses, and the caudal zone native and maladaptive synapses (for the right side of the brain as shown in Fig. 1; the layout on the left side is reversed). How are these functionally independent circuits optimized? Here, we demonstrate that axodendritic contacts were spaced substantially closer together in the adaptive zone than in either normal or maladaptive zones. At the same time, the number of contacts per branch was similar across zones. To gain insights into how this arrangement arose during learning, we examined clustering in juvenile owls at the age of prism attachment. In this group, the spacing between contacts also varied with topographic location, but displayed a distinct pattern, with the highest clustering observed in the normal zone. Collectively, these results provide the first direct evidence in support of experience-driven contact clustering as a mechanism for learning.

\section{Materials and Methods}

Animals. Sixteen barn owls (Tyto alba) were used in this study. Animals were housed in group aviaries and cared for in accordance with the University of California, Davis, animal care policy.

Prism rearing. Six newly fledged owls received fresnel lenses with $19^{\circ}$ lateral displacement (prisms). Surgical attachment of headgear was performed at 60-70 d after which the owls were returned to flight aviaries where they hunted live mice and interacted with other owls. This natural experience drives learning, which manifests as an adaptive change in orienting behavior dependent on plasticity of the auditory space map located in the midbrain nucleus, the ICX. The anatomical, physiological, and behavioral changes induced by prism experience typically develop over $60 \mathrm{~d}$. The duration of prism experience for the owls used here ranged from 80 to $210 \mathrm{~d}$. Details of the surgical procedure and conditions of prism rearing can be found in the study by Swofford and DeBello (2007).

Electrophysiology. Adaptive shifts in five of the six prism-reared owls were confirmed using electrophysiological mapping. One prism owl was not mapped because the headgear detached in the recording chamber and could not be resecured. The normal juvenile owls $(n=5$; ages, $60-70$ 
A

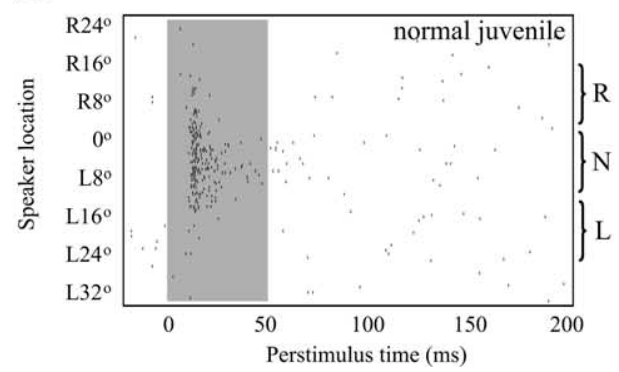

B

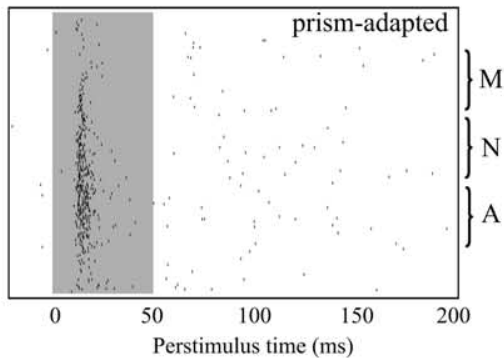

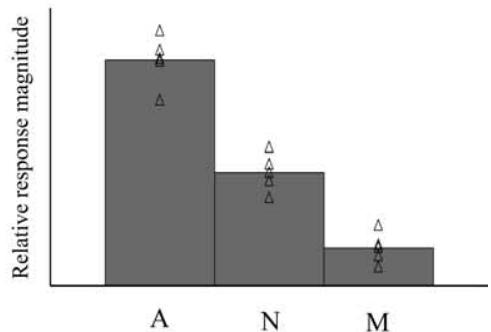

Figure 2. Electrophysiological mapping. $\boldsymbol{A}$, Top, Representative raster plot showing auditory tuning in a normal juvenile. The shaded region indicates duration of sound stimulus. Sound-evoked action potentials are represented as ticks. The visual receptive field at this site was centered at $\mathrm{L} 4^{\circ}$, which predicts range of normal auditory responses (N). Bottom, Representative raster plot from an owl adapted to $\mathrm{R} 19^{\circ}$ prisms. R-shifting prisms require the acquisition of $\mathrm{L}$-ear leading responses. The visual receptive field for this site was centered at $\mathrm{L} 3^{\circ}$. The predicted normal (N) and maladaptive (M) auditory responses are weak, whereas the predicted adaptive responses (A) are strong. $\boldsymbol{B}$, Summary data from five prism-adapted owls that were subsequently analyzed for contact clustering. The triangles represent the relative response magnitudes in each zone for each animal, and the bars indicate the mean across animals.
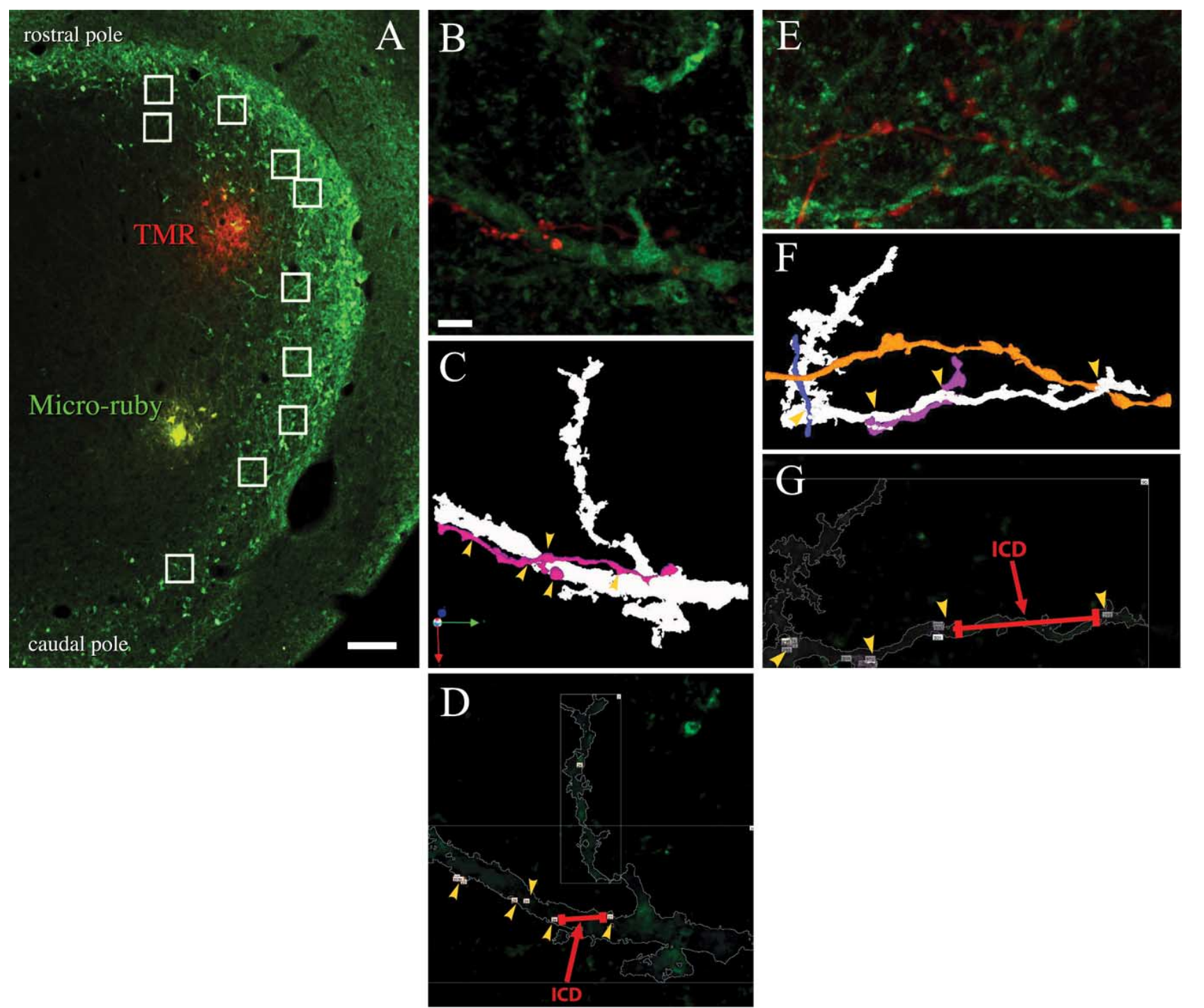

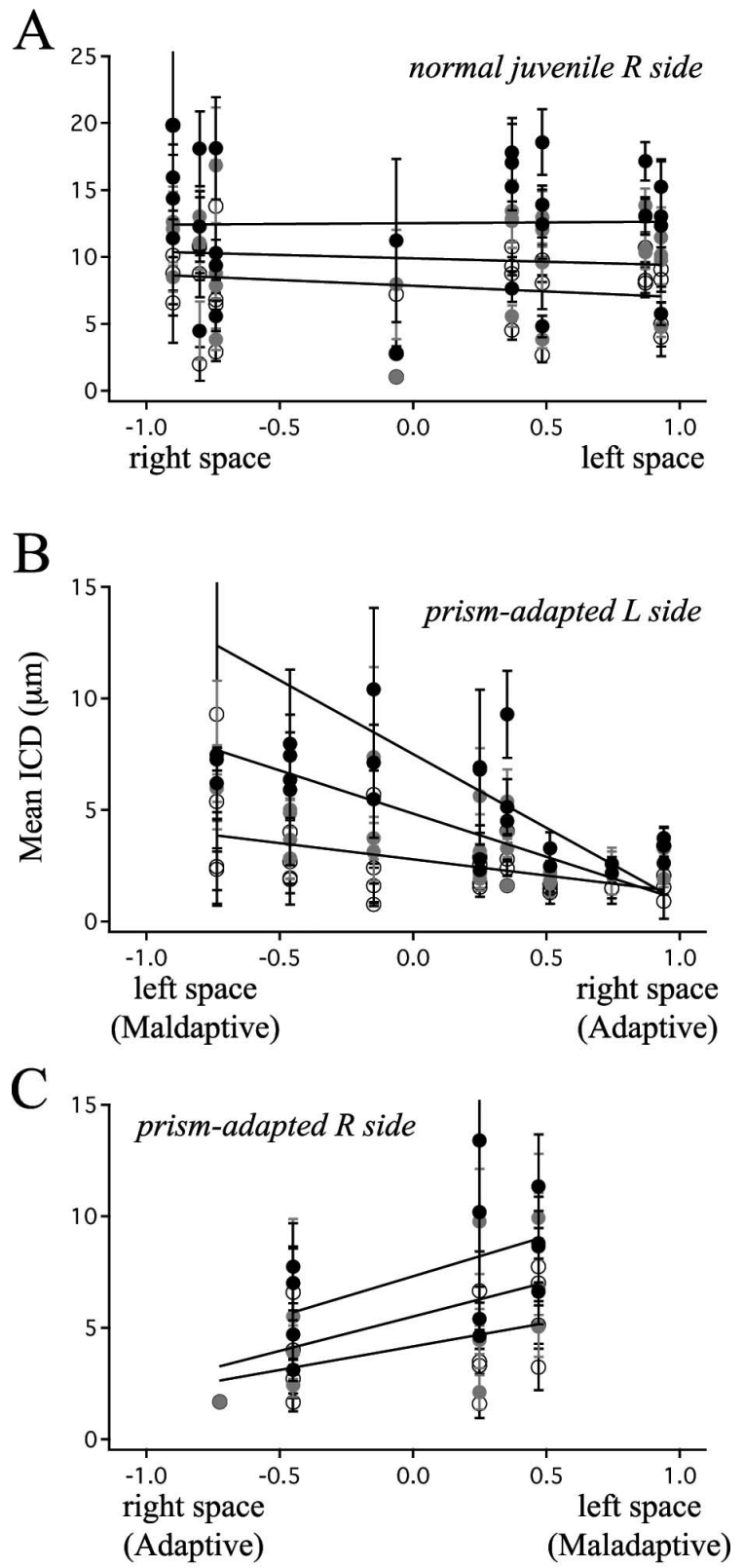

Rostrocaudal location ( $\mathrm{mm}$ )

Figure 4. Contact clustering in normal juvenile and prism-adapted owl: representative cases. $A$, Normal juvenile case, $R$ side of brain. Mean ICD was calculated for each quadrant of each image field $(n=8)$. The rostrocaudal location of the center of each image field, relative to injection site location, is plotted on the abscissa. There were four or fewer quadrants per location because not all quadrants contained dendrites with multiple contacts for which an ICD could be measured. The open circles represent ICD measurements based on aggregate size of 0.5 $\mu \mathrm{m}$; gray circles, aggregate size of $1.0 \mu \mathrm{m}$; black circles, aggregate size of $2.0 \mu \mathrm{m}$. Error bars are SEM. Lines are linear regressions for each dataset. The correlations were near zero regardless of the aggregate size used for analysis ( $0.5 \mu \mathrm{m}$ microcluster size: $n=30$ quadrants, $r=$ $-0.160, p=0.3941 ; 1.0 \mu \mathrm{m}$ microcluster size: $n=30$ quadrants, $r=-0.088, p=0.6439$; $2.0 \mu \mathrm{m}$ microcluster size: $n=29$ quadrants, $r=0.019, p=0.9222)$. B. Prism-adapted case, L side of brain ( $n=8$ image fields). Significantly more clustering was observed in the adaptive zone, which on the $L$ side of the brain is caudal to the injection site. The correlations were strong regardless of the aggregate size used for analysis $(0.5 \mu \mathrm{m}$ microcluster size: $n=29$ quadrants, $r=-0.465, p=0.011 ; 1.0 \mu \mathrm{m}$ microcluster size: $n=27$ quadrants, $r=-0.540, p=$ 0.0036; $2.0 \mu \mathrm{m}$ microcluster size: $n=25$ quadrants, $r=-0.472, p=0.0172)$. C, Prismadapted case, $R$ side of brain ( $n=4$ image fields). Significantly more clustering was observed in the adaptivezone, which on the R side of the brain is rostral to the injection site. The correlations were modest regardless of the aggregate size used for analysis ( $0.5 \mu \mathrm{m}$ microcluster size: $n=$ 13 quadrants, $r=0.437, p=0.1352 ; 1.0 \mu \mathrm{m}$ microcluster size: $n=13$ quadrants, $r=0.495$, $p=0.0858 ; 2.0 \mu \mathrm{m}$ microcluster size: $n=12$ quadrants, $r=0.489, p=0.1066$ ). d) were not mapped because good auditory-visual alignment is reliably established by this age (Feldman and Knudsen, 1997). Data from a single normal juvenile ( $65 \mathrm{~d}$ of age) obtained for a different study are included in Figure 2.

Details of the recording procedure can be found in the study by Swofford and DeBello (2007). Briefly, owls were lightly anesthetized and secured in a stereotax located within a sound-proof room. Multiunit recordings were obtained from the right OT. Auditory stimuli were presented using a free-field speaker array ( $4^{\circ}$ separation), and visual stimuli by hand-held projection onto a calibrated tangent screen. Auditory spatial receptive fields were determined by plotting the response magnitude of each multiunit versus speaker location. Within this receptive field, normal responses were defined as those elicited by speakers within the region predicted by the visual receptive field measured with prisms removed ( $\pm 6^{\circ}$ range centered on best visual receptive field). Adaptive responses were defined as those occurring in the adjacent $12^{\circ}$ region that extended to the edge of the prismatically displaced visual field, and maladaptive responses defined as the $12^{\circ}$ region on the opposite side. These subdivisions of the auditory spatial receptive field correspond on average to the zones in ICX used for microanatomical analysis.

In vitro anterograde tracing. Axons were labeled by focal injection of anterograde tracer into the ICCls of living brain slices. The following two different artificial CSF (ACSF) solutions were prepared: sucrose ACSF (in mM: 240 sucrose, $3 \mathrm{KCl}, 3 \mathrm{MgCl}_{2}, 1.2 \mathrm{NaH}_{2} \mathrm{PO}_{4}, 23 \mathrm{NaHCO}_{3}$, and 11 D-glucose) and normal ACSF (in mM: $120 \mathrm{NaCl}, 3 \mathrm{KCl}, 1 \mathrm{MgCl}_{2}, 2 \mathrm{CaCl}_{2}$, $1.2 \mathrm{NaH}_{2} \mathrm{PO}_{4}, 23 \mathrm{NaHCO}_{3}$, and 11 D-glucose). Both were $\sim 300$ mOsmol, oxygenated with carbogen, $\mathrm{pH}$ 7.4. Animals were anesthetized and briefly perfused with oxygenated ice-cold sucrose ACSF. After the blood cleared, the animals were decapitated and the head opened along the midsagittal plane. The tectal lobes were isolated and maintained in oxygenated ice-cold sucrose ACSF. Horizontal slices $(350 \mu \mathrm{m})$ were prepared with a vibratome (Leica) and kept in oxygenated normal ACSF at room temperature (RT). Glass micropipettes (1.5 mm outer diameter, with filament; WPI) were pulled to a tip diameter of $1-2 \mu \mathrm{m}$ using a Flaming/Brown puller (Sutter Instrument). Glass electrodes were back filled with a solution containing $5 \%$ micro-ruby or Texas Red in $1 \mathrm{M} \mathrm{KCl}$ and positioned using a micromanipulator at the center of a custom-made chamber filled with oxygenated normal ACSF. Slices were put into the chamber, and tracer was applied under visual control using a stereo microscope ( $4 \times$ objective; Carl Zeiss). Continuous current (0.2-0.5 $\mathrm{mA}$ ) was passed through a customized head stage at $7 \mathrm{~s}$ intervals. To allow for anterograde transport, the slices were incubated in carbogen-bubbled normal ACSF for $1-2 \mathrm{~h}$. They were subsequently placed in fixative [ $4 \%$ paraformaldehyde in $0.1 \mathrm{M}$ phosphate buffer $(\mathrm{PB})]$ at $4^{\circ} \mathrm{C}$ overnight and then transferred to $30 \%$ sucrose in $0.1 \mathrm{M} \mathrm{PB}$ at $4^{\circ} \mathrm{C}$ for $24 \mathrm{~h}$. Fixed slices were resectioned at $30-40 \mu \mathrm{m}$ on a freezing microtome, placed in $0.1 \mathrm{M}$ $\mathrm{PB}$, and processed for immunohistochemistry.

Immunohistochemistry. Dendrites were labeled by immunoreactivity for calcium/calmodulin-dependent protein kinase type II, a subunit $(\mathrm{CaMKII} \alpha)$. Sections were incubated in blocking solution ( $4 \%$ normal goat serum; Vector Laboratories), $1 \%$ bovine serum albumin (BSA) (Thermo Fisher Scientific), and 0.4\% Triton X-100) for $1 \mathrm{~h}$ at RT. Primary antibody (mouse anti-CaMKII $\alpha$, clone 6G9, subtype IgG1; Millipore Bioscience Research Reagents) was diluted 1:500 in 1\% normal goat serum, $0.3 \%$ Triton $\mathrm{X}-100$, and $1 \%$ BSA. Tissue was incubated in primary antibody for $48-72 \mathrm{~h}$ at $4^{\circ} \mathrm{C}$ under gentle agitation. After primary antibody incubation, tissue was rinsed two times for $5 \mathrm{~min}$ in $0.02 \%$ Triton X-100 and $0.25 \%$ BSA at RT. Secondary antibodies were diluted at 1:1000 in $0.02 \%$ Triton X-100 and $1 \%$ BSA and incubated at RT for $1-2 \mathrm{~h}$. Before mounting, tissue was rinsed five times for $2 \mathrm{~min}$ in $0.25 \% \mathrm{BSA}$ and two times for $5 \mathrm{~min}$ in $0.1 \mathrm{M} \mathrm{PB}$. Tissue sections were mounted on gelatinized coverslips $\left(0.3 \%\right.$ gelatin in distilled $\left.\mathrm{H}_{2} \mathrm{O}\right)$, allowed to dry for $10-15 \mathrm{~min}$, and inverted and sealed onto slides using Gel Mount (Biomeda). All dilutions were made in $0.1 \mathrm{M} \mathrm{PB}$, except where indicated.

Confocal imaging and digital processing. Imaging was performed on a Zeiss LSM 510 META confocal microscope (Carl Zeiss). High-resolution images were obtained with a $63 \times$ oil-immersion objective (Plan Apochromat; numerical aperture, 1.4; Olympus oil, refractive index 1.515). The confocal aperture was set at 1 airy unit. Image frame size was $1904 \times$ 
1904. Step size for $z$-stacks was $150 \mathrm{~nm}$, resulting in voxel dimensions of 50,50, and $150 \mathrm{~nm}(x, y$, and $z$, respectively). Confocal images were deconvolved using Huygens Professional 2.9 (SVI). To determine point spread functions (PSFs), we imaged subresolution fluorescent beads (TetraSpeck; $0.1 \mathrm{~mm}$ diameter; Invitrogen). Deconvolution was performed using measured PSFs and a classic maximum-likelihood estimation algorithm.

It was not possible to analyze all axons emanating from a single injection site, because the quantitative microanatomical analysis described below was extremely time intensive. Therefore, we developed a strategy for sampling the labeled axons. High-resolution image fields were distributed across the rostrocaudal extent of the arbor (up to $2 \mathrm{~mm}$ ) (for a representative example of the axonal coverage that resulted from this strategy, see Fig. 3A). Field-to-field variations in the density of axons within a single reprocessed section spanned several orders of magnitude, and occurred with a patchy distribution: image fields containing very sparse labeling could be found adjacent to fields containing dense labeling. To minimize the impact of local variations in axonal (or dendritic) neuropil density on clustering measurements, fields for high-resolution images were selected by visual inspection to contain a comparable, medium-high density of axons and a dense plexus of CaMKII staining. Quadrant-toquadrant variations in axonal density were expectedly larger than field-to-field variation. Still $95 \%$ of the data points fell within a fivefold range of axonal densities (data not shown). Quadrantto-quadrant variations in total neuropil density are shown in Figure 9. Field selection and analysis were performed by a case blind observer.

Microanatomical measurements. Image analysis was performed using Volocity 3.0.1 (Improvision) running on a 64 bit dual processor Mac G5. Each deconvolved high-resolution image field was divided into four quadrants for threedimensional reconstruction and analysis because the computational load was otherwise too great. Quadrant size was $48 \times 48 \times 10 \mu \mathrm{m}$. To search for sites of contact between tracer-labeled axons and CaMKII+ neurons, three-dimensional profiles were generated from confocal stacks using a lower threshold intensity value of 10 . Each contiguous axon and dendritic plexus was isolated as a separate object using the classifier tools. Potential sites of contact between axons and dendrites were identified automatically by the presence of double-labeled voxels at their interface, using the overlap tools. These "overlap objects" were classified as axodendrite contacts or bouton-dendrite contacts on the basis of visual inspection. Boutons were identified as swellings no longer than $3 \mu \mathrm{m}$, with a diameter at least 1.5 times greater than the diameter of the adjoining axon. Overlap objects were associated with the individual axons and dendritic branches on which they occurred. The type, size, and location of each contact site, the cognate axon and dendrite branch, and the dimensions of all axons and dendrites were stored as primary data for additional analysis.

Quantitative analysis to determine contact clustering. Primary data were analyzed using custom programs written by W. M. DeBello in IgorPro (Wavemetrics). The strategy was to (1) identify potential sites of synaptic contact by screening the overlap objects and (2) measure the distance between contacts on individual branches of dendrite, referred to as the intercontact distance (ICD). First, to exclude overlap objects represent-

E $p=0.0003)$. normal juveniles
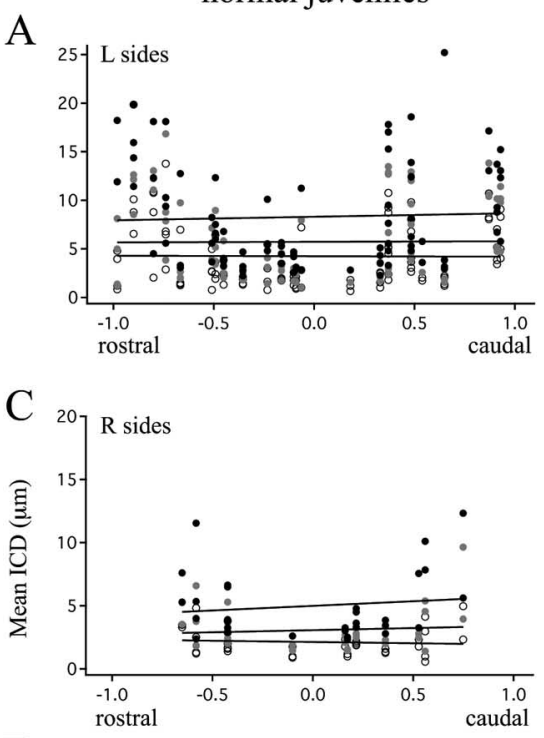

Figure 5. Contact clustering in normal juvenile and prism-adapted owl: su $\mu$ mary data. Data were pooled across 9 injections performed in five normal juvenile owls, and 11 injections in six prism-adapted owls. All details of this presentation are as were near zero (0.5 $\mu \mathrm{m}$ microcluster size: $n=95$ quadrants, $r=0.008, p=0.9414 ; 1.0 \mu \mathrm{m}$ microcluster size: $n=93$

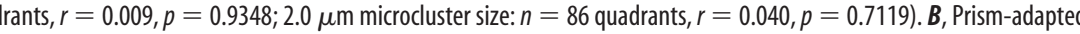
quadrants, $r=-0.279, p=0.0098 ; 1.0 \mu \mathrm{m}$ microcluster size: $n=76$ quadrants, $r=-0.333, p=0.0033 ; 2.0 \mu \mathrm{m}$ (1) $n=66$ quadrants, $r=-0.325, p=0.0077)$. C, Normal juvenile cases, R side of brain. Correlations were near $0.087, p=0.6294: 2.0 \mu \mathrm{m}$ microcluster size $n=30$ quadrants, $r=0.126, p=0.5081)$. D Prism-adapted cases, R side of brain. Correlations were significantly shifted in the adaptive direction ( $0.5 \mu \mathrm{m}$ microcluster size: $n=52$ quadrants, $r=0.564$, $p<0.0001 ; 1.0 \mu \mathrm{m}$ microcluster size: $n=46$ quadrants, $r=0.562, p<0.0001 ; 2.0 \mu \mathrm{m}$ microcluster size: $n=46$ quadrants, $r=0.446, p=0.0031) . E$, All normal juvenile cases, replotted relative to right space/left space by inverting the abscissa values $2.0 \mu \mathrm{m}$, respectively) were better fit by a polynomial function. $\boldsymbol{F}$, All prism-adapted cases, replotted as described. Correlations were significantly shifted in the adaptive direction ( $0.5 \mu \mathrm{m}$ microcluster size: $n=137$ quadrants, $r=0.327, p<0.0001 ; 1.0$ $\mu \mathrm{m}$ microcluster size: $n=122$ quadrants, $r=0.372, p<0.0001 ; 2.0 \mu \mathrm{m}$ microcluster size: $n=108$ quadrants, $r=0.344$,

ing close passes of axon and dendrite not in physical contact, objects were required to meet a minimum size threshold (Rodriguez-Contreras et al., 2005). Objects smaller than 4 voxels were discarded. Next, microclusters of objects that occurred in very close proximity were counted as single contacts. This restriction was accomplished using microcluster size limits of $0.5,1.0$, and $2.0 \mu \mathrm{m}$ as shown in Figures 4 and 5. No qualitative differences were observed and all subsequent analyses were performed using the most conservative definition of contacts $(2.0 \mu \mathrm{m}$ microcluster size). When multiple contacts occurred on the same dendritic branch, the ICDs were determined from the three-dimensional addresses measured automatically by Volocity, as the straight-line distance from one contact to its nearest neighbor (see Fig. 3). Because the dendritic segments analyzed were nearly linear, this value closely approximated the distance between contacts measured along the dendritic path. Finally, the 
A
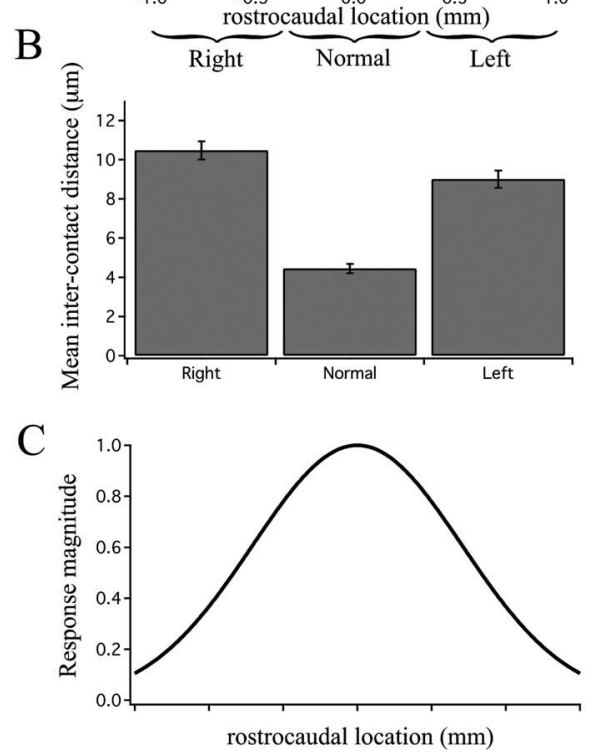

prism-adapted
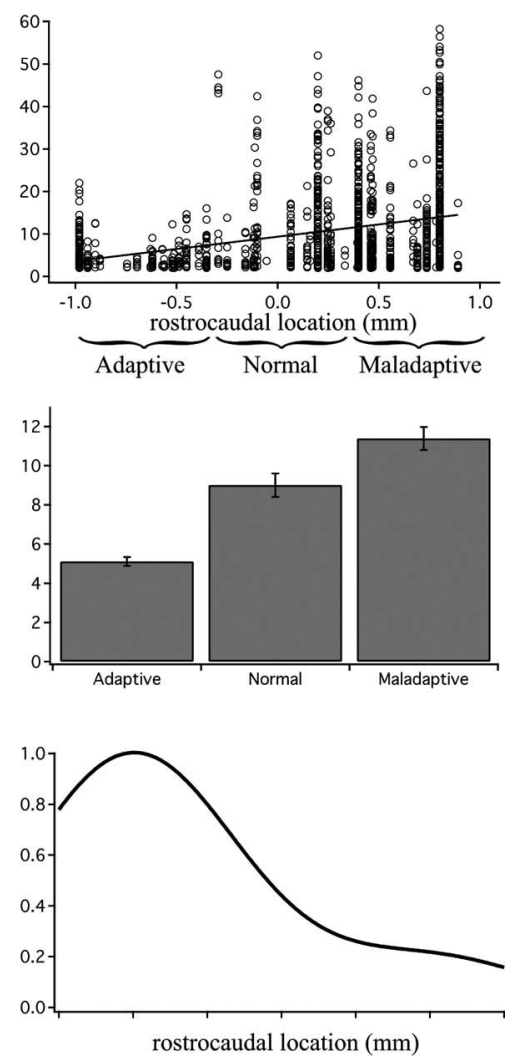

Figure 6. Distributions of ICDs across adaptive, normal, and maladaptive zones and their correspondence with physiological response magnitude. $A$, Left, Distribution of all ICD values ( $n=1733 ; 2.0 \mu \mathrm{m}$ microcluster size) for normal juveniles, plotted as in the bottom panels of Figure 5. Right, Same analysis for prism-adapted owls ( $n=1331$ ICDs; $r=0.278 ; p<0.0001)$. $\boldsymbol{B}$, Data were divided into three equal zones based on rostrocaudal location. The mean values for each zone are shown for normal juveniles (left) and prism-adapted owls (right). Significant differences were determined by unpaired Student's $t$ test (Table 1). Error bars indicate SEM. C, Stylized tuning curves indicate the response profile characteristic of each microanatomical zones [based on Gold et al. (2000) and DeBello et al. (2001).

amount of axonal or dendritic neuropil was taken as the automated reported value for the surface area of classified objects. Statistical analyses were performed using Igor or StatView (SAS Institute). Values reported for all linear regressions are as follows: $n$, number of data points; $r$, Pearson's correlation coefficient; $p, p$ value based on standard $r$ to $p$ conversion.

The rostrocaudal location of each high-resolution image quadrant was determined from low-magnification images obtained on a Zeiss Axioscop 2 FS (Carl Zeiss) with a $5 \times$ objective. A contour delineating the auditory space map in ICX was made by tracing a curved line through the region of highest CaMKII intensity, from rostral to caudal pole. A radial projection from the center of the injection site in ICCls was traced to this ICX contour, and the location of each image quadrant was measured as the distance along the ICX contour between the center of its parent image field (outlined by modest photobleaching that had occurred during acquisition) and the projected location of the injection site.

Homer 1 and gephryin analysis. In vitro slices were prepared from a naive owl and tracer injections placed. To maximize detection of postsynaptic proteins, slices were lightly fixed by immersion in $2 \%$ paraformaldehyde for $10 \mathrm{~min}$ (Schneider Gasser et al., 2006), cryoprotected, and resectioned. CaMKII was detected with Alexa Fluor 647 secondary. Homer 1 or gephryin were detected in adjacent sections using rabbit polyclonal antisera (Synaptic Systems) and Alexa Fluor 488 secondary. High-resolution images were acquired and deconvolved as previously described. Homer 1 or gephryin punctae were classified by coapplication of size $\left(0.037 \mu \mathrm{m}^{3}\right.$ minimum) and intensity (Homer 1, $10 \mathrm{U}$; gephryin, $20 \mathrm{U})$ thresholds. Axodendritic contacts between tracer (red channel) and CaMKII+ dendrites (far-red channel displayed in blue) were iden- tified as previously described. Contacts were counted as positive or negative for Homer 1 or gephryin based on the presence or absence of volume overlap with classified synaptic punctae, determined automatically using the session arithmetic functions in Volocity.

Monte Carlo simulations. To assess the impact of false-positive contacts on the observed differences in ICD between adaptive (A) and maladaptive (M) flanks, we used a stochastic elimination process. Dendrite by dendrite, contacts were flagged by random sorting without replacement according to a fixed probability (the false-positive rate), and then eliminated. Some dendrites were untouched, and others heavily winnowed. After contact elimination, the number of contacts, mean ICD, and $p$ value (Student's $t$ test) for differences between the simulated $\mathrm{A}$ and $\mathrm{M}$ datasets were calculated. False-positive rates of 20, 40, 60, 80, and 90\% were examined. Each was applied to the primary data on five independent trials to create five simulated datasets for A and $M$, respectively. This yielded 25 statistical comparisons for each of the five false-positive rates (see Fig. $12 \mathrm{~F})$. Simulations, curve fitting, and statistical analysis were performed in IgorPro.

\section{Results}

The goals of this study were (1) to measure the spatial clustering of axodendritic contacts along individual branches of dendrites, (2) to determine whether clustering varies with topographic location across the coactive axonal arbors that provide synaptic input, and (3) to determine whether the clustering patterns change with learning. To quantify functional changes in circuit strength, prism-reared owls were analyzed by electrophysiological recording before microanatomical analysis. Data were compared with those from a second experimental group, normal juveniles, whose brains represent the initial state of the circuit.

\section{Adaptive adjustments in auditory tuning in prism-adapted owls}

Six owls were raised from $\sim 70 \mathrm{~d}$ of age wearing prisms that shifted the visual field $19^{\circ}$ to the right. Adaptive adjustments were confirmed using multiunit recording from the deep layers of optic tectum (OT). A representative auditory spatial tuning curve recorded from a prism-adapted owl (Fig. $2 A$ ) demonstrates the expression of strong adaptive responses, corresponding to the optically displaced visual field location, weakened normal responses corresponding to the visual field location with prisms removed, and weak maladaptive responses on the opposite flank of the tuning curve. The mean response magnitudes for each zone are shown in Figure $2 B$. A representative example of auditory tuning recorded from a normal juvenile (Fig. $2 A$ ) demonstrates the expression of strong normal responses and weak responses on either flank, which by definition are maladaptive. These regions of the tuning curve in prism-reared owls (adaptive, normal, maladaptive) and normal juveniles (right space, normal, left space) are physiological correlates of the microcircuit zones in ICX that were analyzed for contact clustering. 
Visualization and analysis of axodendritic contacts in ICX

Axons were labeled by focal injections of anterograde tracer, micro-ruby or Texas Red dextran amine, into the ICCls of living brain slices. Injections were placed at locations representing between $0^{\circ}$ and contralateral $20^{\circ}$ of auditory space (Fig. 3), map locations that reliably undergo physiological and anatomical plasticity. The terminating fields of the labeled axonal arbors were topographically restricted within ICX, and qualitatively indistinguishable from the terminal fields labeled by in vivo tracer injections (DeBello et al., 2001; Linkenhoker et al., 2005). It is within these broad arbors that adaptive, normal, and maladaptive zones were distinguished on the basis of rostrocaudal position relative to injection site location.

Postsynaptic targets for ICCls axons include space-specific neurons, a major class of which expresses CaMKII throughout their entire somatodendritic architecture (Rodriguez-Contreras et al., 2005). We analyzed the spatial pattern of contacts between tracer-labeled ICCls axons and CaMKII+ dendrites in highresolution confocal image fields distributed along the rostrocaudal extent of the arbor (Fig. 3A). Two representative examples of threedimensional reconstructions and methods for analysis are shown in Figure 3, B-G.

\section{Contact clustering in prism-adapted and normal juvenile owls}

Axodendritic contacts (potential synapses) were identified based on strict criteria defined in Materials and Methods. We focused analysis on two features directly relevant to the hypothesis, contact clustering and number of contacts per segment of dendrite. Contact clustering was determined by measuring the distance between contacts on dendritic segments that received more than one contact. Lower values of intercontact distance, therefore, reflect a higher degree of clustering. Data for a representative normal juvenile case are shown in Figure $4 A$. Linear regression uncovered no systematic rostrocaudal asymmetry. These data were better fit by a U-shaped, polynomial function (Figs. 5E, 6A) that revealed greater clustering in the central, normal zone of the arbor compared with either rostral or caudal flanks, representing right and left space, respectively. This U-shape pattern mimics the spatial pattern of auditory tuning characteristic of normal juveniles (Fig. 6C).

Data for a representative prism-adapted case are shown in Figure $4, B$ and $C$. In contrast to the results for the normal juvenile, linear regression revealed a significant correlation between contact clustering and rostrocaudal location: On the left (L) side of the brain, the slope of the regression was negative, indicating greater contact clustering on the caudal flank. The caudal flank represents adaptive space on the $\mathrm{L}$ side. On the right $(\mathrm{R})$ side of the brain, the slope of the regression was positive, indicating greater clustering on the rostral flank. The rostral flank represents adaptive space on the $\mathrm{R}$ side. This rostrocaudal asymmetry in clustering cannot be explained as normal developmental progression, but instead reflected the asymmetry imposed by prisms (Fig. 1).

The effects described above were robust and observed in data pooled across individuals (Fig. 5). To combine data from both sides of the brain for subsequent analysis, the rostrocaudal axis for $\mathrm{L}$ side cases was flipped and the data were pooled (Figs. $5 E, F$, $6,7,9,10)$. This transforms the rostrocaudal axis into an adaptive-maladaptive axis (see figure legend). Data pooled in this way were subdivided into three equal regions representing, for prismadapted owls, the adaptive, normal, and maladaptive zones, and
Table 1. $p$ values for comparisons between all zones (unpaired Student's $t$ test)
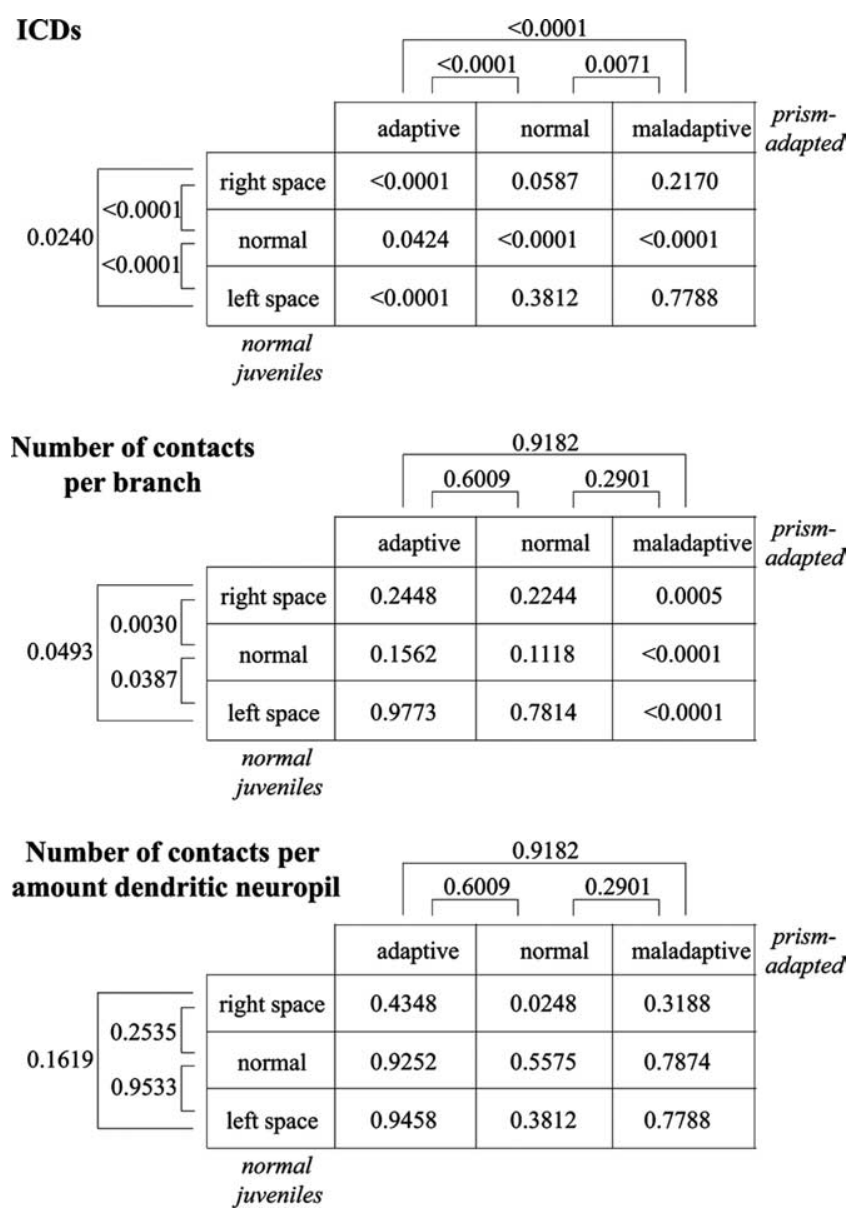

for normal juveniles, comparable zones representing right space, normal, and left space.

\section{Distributions of intercontact distances and microanatomical density of contacts}

The distribution of intercontact distances across zones is shown in Figure $6 A$, and the mean values for each zone in Figure $6 B$. In normal juveniles, clustering was significantly higher in the normal zone (mean ICD, $4.4 \pm 0.24 \mu \mathrm{m}$ ), than in either flanking zones (right space, mean ICD, $10.5 \pm 0.47 \mu \mathrm{m}$; left space, mean ICD, $9.0 \pm 0.44)$. In contrast, in prism-adapted owls clustering was highest in the adaptive zone (mean ICD, $5.1 \pm 0.23 \mu \mathrm{m}$ ), intermediate in the normal zone $(9.0 \pm 0.60 \mu \mathrm{m})$, and lowest in the maladaptive zone (mean ICD, $11.4 \pm 0.58 \mu \mathrm{m}$ ). Collectively, these data demonstrate two distinct prism-induced effects: a large, approximately twofold increase in clustering on the adaptive flank (compare the adaptive zone in prism owls with the corresponding right space zone in normal juveniles) and a comparably sized decrease in clustering in the normal zones. These clustering patterns mirrored the functional strengths of the corresponding microcircuits, represented as stylized tuning curves in Figure 6C.

We next focused on two measures of microanatomical contact density: the number of contacts per branch and the number of contacts per amount of dendritic neuropil (Fig. 7). In contrast to the results for contact clustering, the number of contacts per branch was similar across all zones, ranging from a minimum of 1.18 in the normal zone of normal juveniles to a maximum of 1.54 in the maladaptive zone of prism owls. More significant, 
there were no prism-induced changes evident for either the adaptive zone (right space zone of normal juveniles, mean, $1.31 \pm 0.03$; vs adaptive zone of prism owls, mean, $1.24 \pm 0.05$ ) or normal zone (normal juveniles, mean, $1.18 \pm 0.02$; prism-adapted, $1.25 \pm 0.04)$. In addition, the number of contacts per amount of dendritic neuropil were unchanged in these zones (Fig. 7C,D). Thus, the changes in contact clustering described above were not driven by adjustments in microanatomical density of contacts, but reflected topographically specific changes in dendritic addressing.

To gain insight into how the differences in contact clustering between the adaptive and maladaptive zones arose, we analyzed the frequency distributions of ICDs (Fig. 8). The ICD distribution for the maladaptive zone was shifted to the right compared with the distribution for the adaptive zone (Fig. $8 \mathrm{~A}$ ), as expected from the difference in mean ICDs, and this difference was highly significant. Shorter ICDs of $20 \mu \mathrm{m}$ or less were found in both distributions, and within this range the maladaptive distribution was shifted to the right. More striking was the complete absence of ICDs $>20 \mu \mathrm{m}$ in the adaptive zone. Widely spaced contacts are not expected to drive postsynaptic action potentials as effectively as more closely spaced contacts (because of nonlinear summation). Their preservation in the maladaptive zone is a microanatomical correlate of the low response magnitude observed during electrophysiological recording (Fig. 2, bottom).

The ICD distribution for normal juveniles represents the initial pattern of clustering. Comparison of the right space zone of normal juveniles with the corresponding adaptive zone in prism owls revealed that all long-range contacts $(>20$ $\mu \mathrm{m}$ ) had been eliminated during prism adaptation (Fig. $8 B$ ). Conversely, comparison of the normal zones in each experimental group revealed that long-range contacts appeared during adaptation (Fig. 8C). These results can only be explained by the operation of a dynamic process (addition and/or subtraction of contacts), with differential selective pressure for long-range contacts in each zone. Finally, comparison of the left space zone of normal juveniles with the corresponding maladaptive zone in prism owl revealed similar prevalence of long-range contacts (Fig. $8 \mathrm{D}$ ).

Relationship between contact clustering and neuropil density In principle, the observed differences in contact clustering could reflect differences in the bulk spatial density of axonal and dendritic neuropil in adaptive versus maladaptive zones. To investigate the relationship between contact clustering and neuropil density, we plotted these values for each image field using data from all cases. There was a weak negative correlation (Fig. 9A),
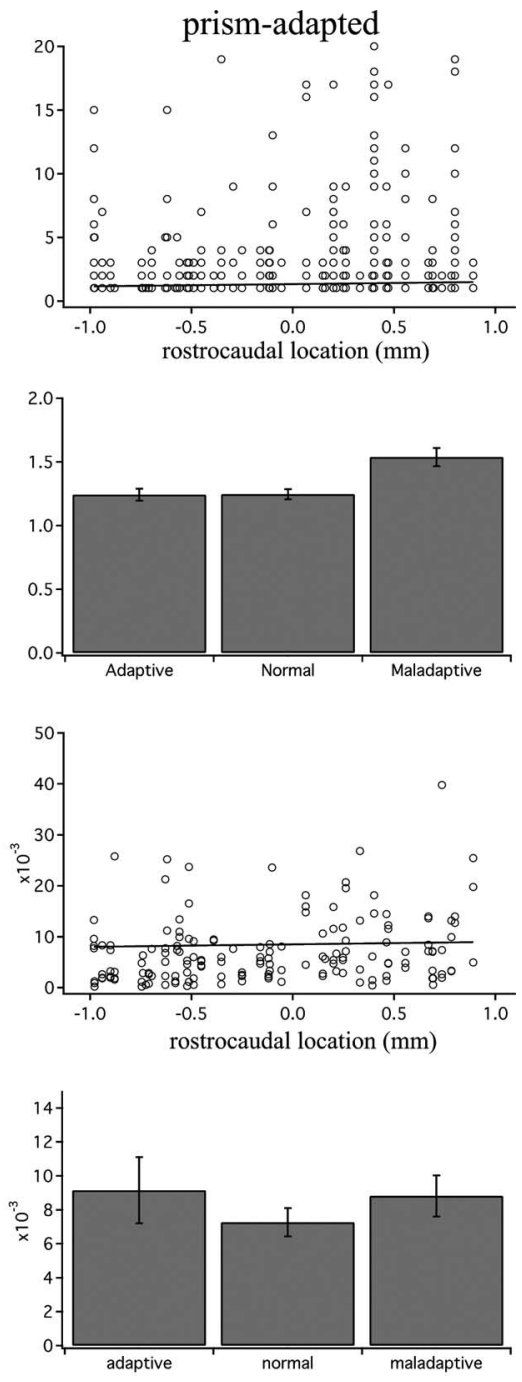

Figure 7. Microanatomical density of contacts. $\boldsymbol{A}$, Number of contacts per dendritic branch versus location for normal 019). The lines are linear regression. $\boldsymbol{B}$, Mean values for each zone. Significant differences were determined by unpaired (Table 1). C, Number of contacts per amount of CaMKII + dendritic neuropil versus location for normal juveniles $22 ; p=0.7768$ ). The lines are linear regression. $\boldsymbol{D}$, Mean values for each zone. Significant differences were determined by unpaired Student's $t$ test (Table 1). Error bars indicate SEM.

indicating that regions of high neuropil density tended to exhibit slightly less contact clustering than regions of lower density. This effect cannot explain the prism-induced changes, however, because neuropil density did not systematically vary with rostrocaudal location for either prism-adapted (Fig. 9B) or normal juvenile owls (Fig. 9C).

\section{Bouton-dendrite contacts}

We examined the subset of axodendritic contacts that were formed by a tracer-labeled presynaptic bouton, including both en passant and terminal varicosities. As expected, the range of values was somewhat larger than the analysis based on the entire cohort of axodendritic contacts, because some branches received widely spaced bouton-dendrite contacts that were intercalated by axodendrite contacts (the latter are absent from the data presented in Fig. 10). For prism-adapted owls, there was a positive correlation between bouton-dendrite ICDs and the adaptive-maladaptive axis, with the greatest 
A

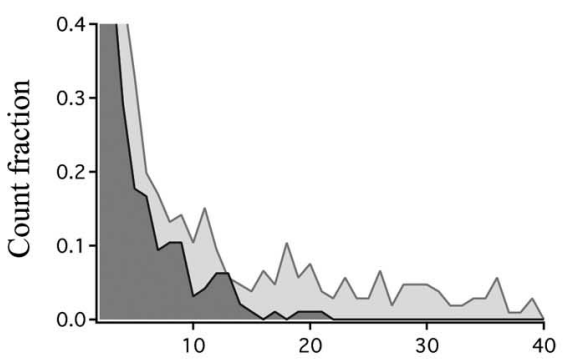

C

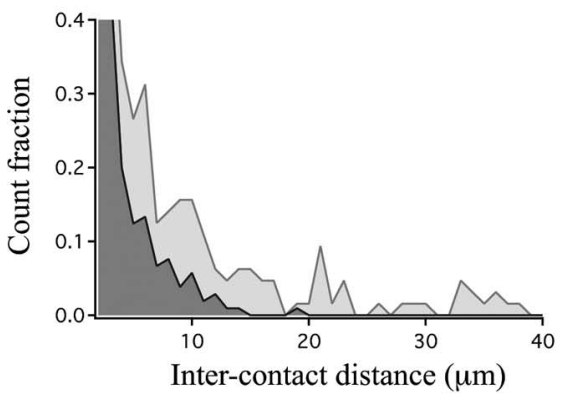

B

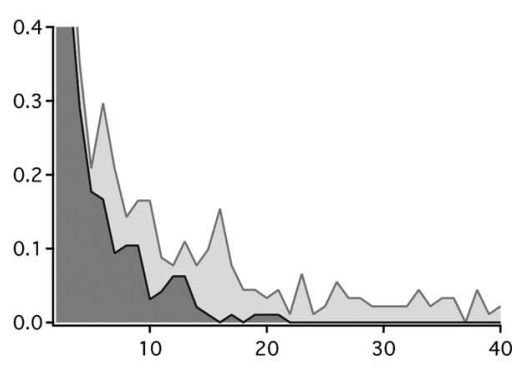

$\mathrm{D}$

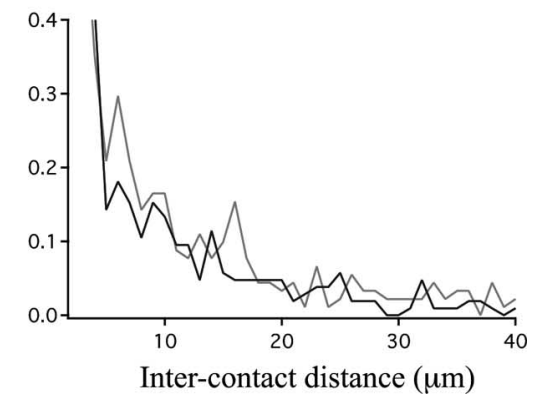

Figure 8. Frequency histograms of ICDs across adaptive, normal, and maladaptive zones. Frequency distributions of ICDs for each zone were normalized to peak, which occurred in the first bin (ICD, 2-3 $\mu \mathrm{m})$ for all zones. $\boldsymbol{A}$, Light gray, Maladaptive zone of prism owls; dark gray, adaptive zone of prism owls ( $n=426$ and $258 \mathrm{ICD}$, respectively; $p<0.0001)$. $\boldsymbol{B}$, Light gray, Right space zone of normal juveniles; dark gray, adaptive zone of prism owls ( $n=485$ and 258 ICDs, respectively; $p<0.0001)$. C, Light gray, Normal zone of prism owls; dark gray, normal zone of normal juveniles ( $n=261$ and 235 ICDs, respectively; $p<0.0001)$. D, Light gray line, Maladaptive zone of prism owls; black line, left space zone of normal juveniles ( $n=426$ and 418 ICDs, respectively; $p<$ 0.0176). The $p$ values were determined by the Kolmogorov-Smirnov test.

clustering observed in the adaptive zone. In contrast, no correlation was observed for normal juveniles. These results recapitulate the main finding of the previous analyses.

\section{Expression of Homer 1 and gephryin at sites of axodendritic contact}

We next asked whether axodendritic contacts are specifically associated with markers of excitatory transmission. Colabeling with antibodies directed against both presynaptic and postsynaptic proteins was complicated by the unique fixation conditions required to optimally visualize tracer, soluble protein (CaMKII) and membrane-bound or scaffolding proteins (Schneider Gasser et al., 2006). Still, we found that antibodies directed against the postsynaptic proteins Homer 1 and gephryin were highly compatible with our microanatomical procedures. Homer 1 is associated with excitatory receptors (Sala et al., 2001; Lu et al., 2007), and gephryin with inhibitory receptors (Schneider Gasser et al., 2006). Both antibodies predominantly labeled synapse-sized punctae (Fig. 11A), which occurred at comparable spatial densities throughout the ICX (Fig. 12A,B). Gephryin punctae $(n=$ $10,199)$ were $10 \%$ more abundant than Homer 1 punctae $(n=$ 15,260) (3.7 per $100 \mu \mathrm{m}^{3}$ vs 3.3 per $100 \mu \mathrm{m}^{3}$ ), whereas the fractional volume of Homer 1 punctae was $41 \%$ greater (1.65 vs $2.80 \%$ ).

The axodendritic contacts described in this study are thought to be predominantly or exclusively excitatory on the basis of physiological recordings and presynaptic and postsynaptic CaMKII expression (Rodriguez-Contreras et al., 2005). Consistent with this expectation, contacts were far more likely to express Homer 1 than gephryin (58.5 vs 17.8\%) (Fig. 12C). Moreover, the overlap objects between axon and dendrite that define sites of contacts were frequently observed to subsume Homer 1 punctae

(Fig. 11C,D). This unique association of contacts with excitatory, and not inhibitory, synaptic markers is additional evidence supporting the correspondence between contacts and synapses.

\section{Monte Carlo simulations}

The Homer 1 results indicate that $\sim 59 \%$ of contacts fulfilled all criteria applied for true synapses, whereas $\sim 41 \%$ did not, an estimate of the false-positive rate. To reveal the impact of false positives on the main conclusions of this study, we performed Monte Carlo simulations. Using the ICD data from the adaptive and maladaptive flanks of prism-adapted owls, a fixed percentage of contacts were randomly eliminated. Intercontact distances were recalculated on the simulated datasets (Fig. 12D$F)$. Our main conclusions remain statistically significant until fully $71 \%$ of the contacts are eliminated, indicating that a true-positive rate of $29 \%$ is acceptable. This critical threshold was well exceeded by the empirical data for Homer 1 colocalization.

\section{Discussion}

This study provides the first demonstration of changes in the clustering of axodendritic contacts as a substrate for learning. The ICCls-CaMKII+ clustering patterns revealed by our analysis were highly specific in terms of topography: terminal zones driving the strongest physiological responses exhibited the greatest amount of clustering, and the ones driving the weakest, the least. This robust correlation between microanatomical configuration and functional circuit strength suggests a model in which postsynaptic potentials caused by closely spaced contacts $(<20 \mu \mathrm{m})$ interact synergistically within the dendrite to drive strong postsynaptic responses, whereas declustered contacts (spaced $>20 \mu \mathrm{m}$ ) drive weaker responses because of less synergism. The discussion that follows addresses the data and assumptions underlying this model, and its implications for the dynamics of synaptic growth and elimination.

\section{Correspondence between contacts and synapses}

The most provocative implications of our data depend on partial correspondence $(>29 \%)$ between axodendritic contacts and synapses. Four lines of evidence support this interpretation. First, the axons and dendrites we studied are known to be connected through a profuse excitatory monosynaptic pathway. This has been shown by analysis of response latencies recorded in vivo (Gold and Knudsen, 2000) and by doublelabel immunoelectron microscopy (Rodriguez-Contreras et al., 2005). Second, we applied an interface thickness criterion that required significant volume overlap between axon and dendrite (Rodriguez-Contreras et al., 2005). Application of this criterion rejected thousands of apparent touches from the dataset, restricting the identification of contacts to those in physical contact. Third, the majority of contacts defined in this manner (59\%) expressed Homer 1, a postsynaptic adaptor protein that selectively marks excitatory synapses. This colo- 


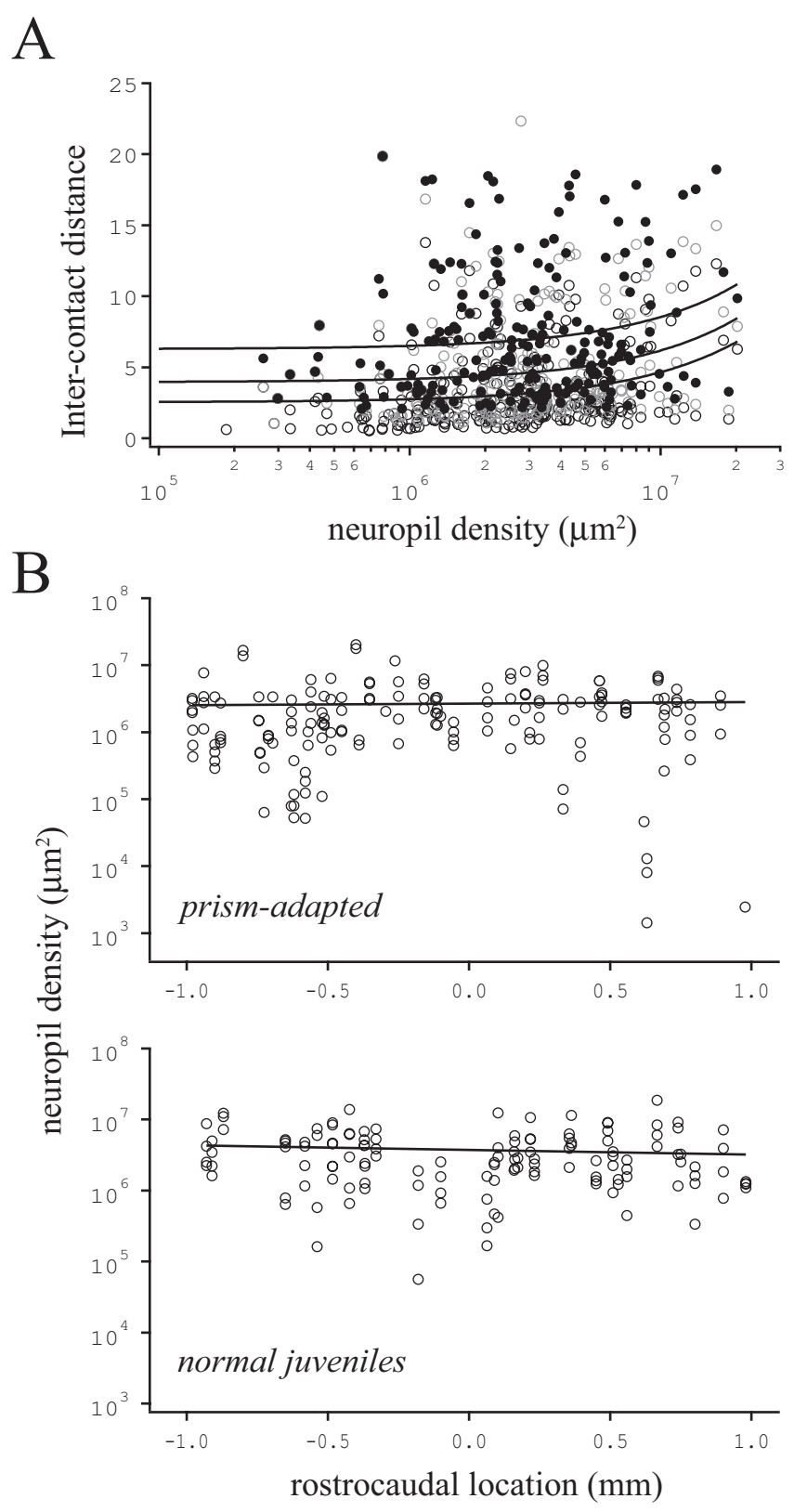

Figure 9. Neuropil density and clustering. $A$, Relationship between ICD and neuropil density for all cases (normal juveniles plus prism-adapted owls). Neuropil density is the product of axonal surface area and CaMKII + surface area for each image quadrant. The open circles represent ICD measurements based on aggregate size of $0.5 \mu \mathrm{m}$; gray circles, aggregate size of 1.0 $\mu \mathrm{m}$; black circles, aggregate size of $2.0 \mu \mathrm{m}$. The lines are linear regressions, which appear curved when displayed on a log scale. There was a slight tendency toward greater clustering in image quadrants with lower neuropil density $(0.5 \mu \mathrm{m}$ microcluster size: $n=266, r=0.236$, $p<0.0001 ; 1.0 \mu \mathrm{m}$ microcluster size: $n=248, r=0.195, p<0.0020 ; 2.0 \mu \mathrm{m}$ microcluster size: $n=224, r=0.021, p<0.0293$ ). $\boldsymbol{B}$, Relationship between neuropil density and rostrocaudal location for prism-adapted (middle) and normal juveniles (bottom). The correlations were near zero (prism-adapted: $n=167, r=0.024, p=0.7587$; normal juveniles: $n=134$, $r=-0.094, p=0.2794$ ). The correlations for regressions based solely on densities of axonal or dendritic neuropil were as follows: prism-adapted, axonal density versus rostrocaudal location, $r=0.099, p=0.2040$; CaMKII density versus rostrocaudal location, $r=-0.031, p=$ 0.6947; normal juveniles, axonal density versus rostrocaudal location, $r=-0.093, p=$ 0.2841; CaMKII density versus rostrocaudal location, $r=-0.114, p=0.1900$.

calization with postsynaptic protein was specific, because gephryin, a marker of inhibitory synapses, was mostly excluded from sites of contact (Fig. 12). Fourth, in one analysis, we restricted definition of contacts to those formed between a

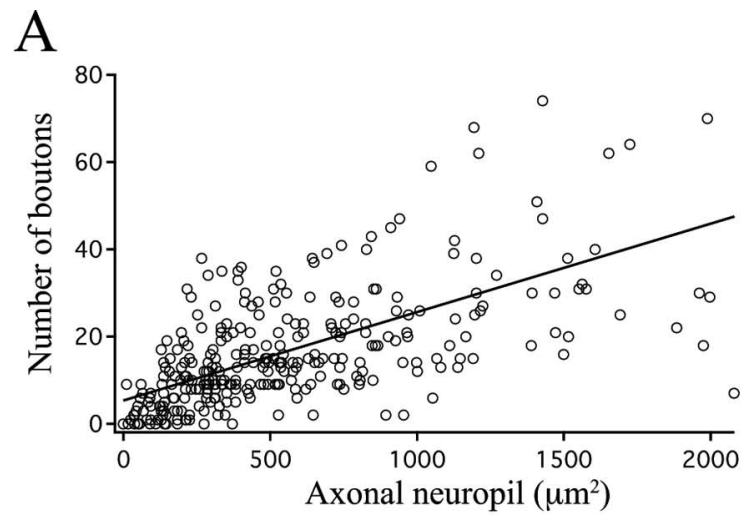

B

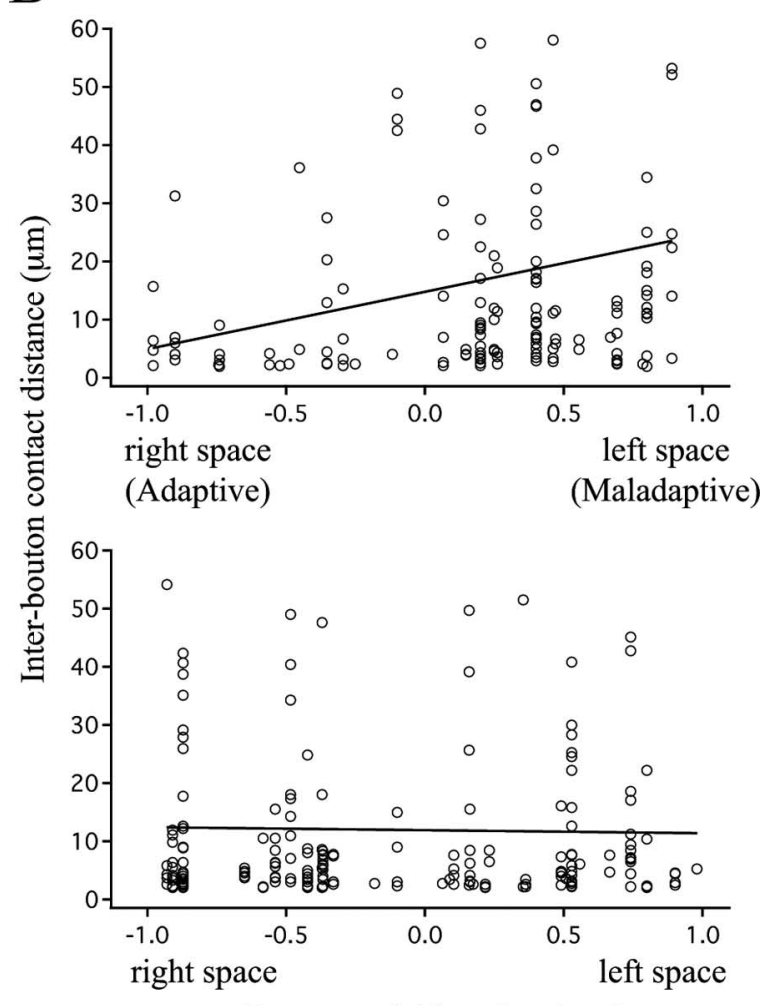

Rostrocaudal location (mm)

Figure 10. Bouton-dendrite contacts. $\boldsymbol{A}$, Relationship between number of boutons and density of axonal neuropil for all cases (normal juveniles plus prism-adapted owls). There was strong positive correlation $(n=301 ; r=0.605 ; p<0.0001)$. B , Distribution of ICD values for bouton-dendrite contacts across the adaptive, normal, and maladaptive zones. This is a recapitulation of the analysis shown in Figure $6 A$, but restricted to contacts formed by a presynaptic bouton. There was a significance correlation for prism-adapted owls $(n=152 ; r=0.220 ; p=$ $0.0065)$, with greater clustering in the adaptive zone. The regression for normal juveniles was nearly flat $(n=205 ; r=-0.018 ; p=0.80)$.

bouton and dendrite (Fig. 10). Such contacts, identified even without the above criteria, show a strong positive correlation with functional synapses as demonstrated by whole-cell recordings in neocortical pyramidal cells (Le Be and Markram, 2006).

The proportion of contacts that represent true synapses is unclear, and could be lower or higher than the 59\% that were Homer $1+$. Some Homer $1+$ contacts are likely to be false positives corresponding to the coincidental aggregation of synaptic protein near sites of contact. The relative failure of gephryin to label contacts, despite a comparable spatial density of gephryin + 

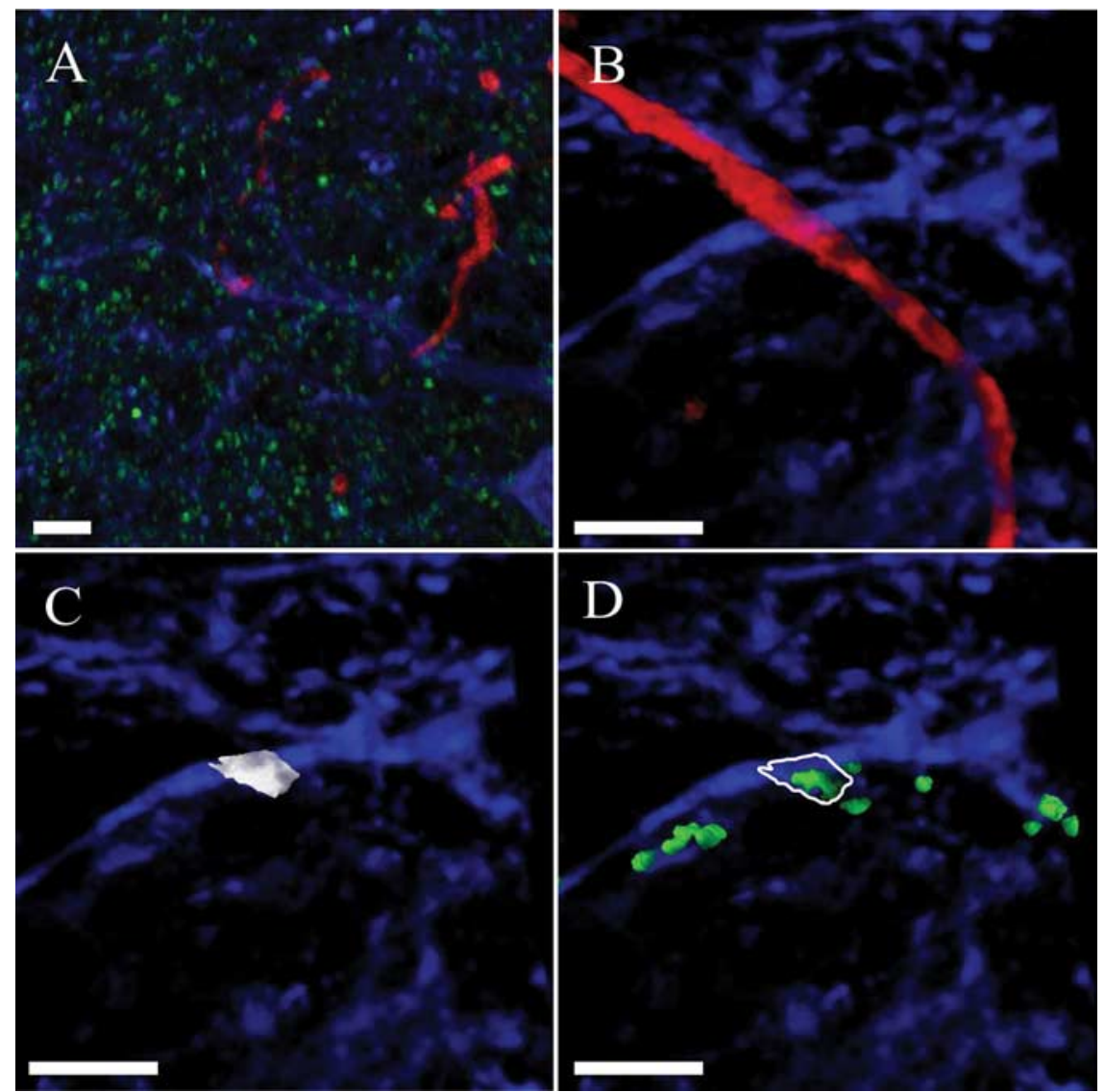

Figure 11. Colocalization of Homer 1 at sites of axodendritic contact. $A$, Triple-labeled image field ( $63 \times$ objective) showing Homer 1 punctae (green), tracer-labeled axons (red), and CaMKII + dendrites (blue). $\boldsymbol{B}$, Image quadrant, rotated and zoomed on an axodendritic contact. Homer 1 punctae were removed for clarity. $C$, Same image with the axon also removed and the overlap volume highlighted in white. $\boldsymbol{D}$, All Homer 1 punctae in contact with the dendritic branch receiving the axodendritic contact are now shown (green). The overlap object (outlined in white) subsumed a synapse-sized punctae. Scale bars: $\mathbf{A}-\mathbf{D}, 5 \mu \mathrm{m}$.

punctae, suggests this fraction is small. In addition, some Homer 1 - contacts are likely to be false negatives representing either neurochemically distinct synapses or sporadic failures in immunohistochemical detection. The latter is typical for proteins of the postsynaptic density (Schneider Gasser et al., 2006) and, in our experience, for presynaptic proteins labeled in the context of intracellular tracers (data not shown).

Functional significance of contact clustering and declustering The physiological correlate of the clustering patterns is the auditory tuning curve. We have shown that clustering is highest where responses are strongest, and this describes the symmetric tuning curves observed in normal juveniles and the asymmetric curves observed in prism-adapted owls. This strong correspondence between auditory-driven firing rate (Fig. 2) and the magnitude of clustering (Fig. 6) suggests a causal relationship.

Whole-cell recordings from neocortical pyramidal cells indicate that the spacing between synapses located on the same branch of dendrite can have a profound effect on the linearity of summation (Polsky et al., 2004). EPSPs caused by synapses located $>80 \mu \mathrm{m}$ from one another sum nearly linearly at the cell body. In contrast, EPSPs located progressively closer together on the dendrite are expected to interact supralinearly. The most significant gains in this functional synergism occur across ICDs of $\sim 40 \mu \mathrm{m}$, whereas additional gains are predicted for synapses even closer together, up to $20 \mu \mathrm{m}$, the shortest intercontact distance analyzed. The prediction of these results is that the functional impact of learning-driven clustering/declustering is maximal for transitions between ICDs greater than $\sim 40 \mu \mathrm{m}$ and ICDs less than $\sim 40 \mu \mathrm{m}$.

The most striking differences in the ICD distributions we measured occurred above and below $20 \mu \mathrm{m}$, slightly smaller than the operating range indicated by the experiments described above. There are at least three nonexclusive interpretations: (1) The integrative properties of CaMKII+ dendrites operate over a more narrow spatial range than the pyramidal dendrites studied. (2) The functional significance of clustering lies not in the electrical properties of CaMKII+ dendrite, but in their capacity for biochemical integration. For example, nonlinear summation of synaptically induced intracellular calcium signals (Augustine et al., 2003). (3) The imperfect correspondence between contacts and functional synapses, specifically the presence of false-positive contacts, resulted in an underestimate of the true ICD values. This explanation cannot, however, account for the learning-driven differences between the adaptive, normal, and maladaptive zones. Finally, it should be noted that the size of the image quadrants used for microanatomical reconstruction limited detection of ICDs to those $<68 \mu \mathrm{m}$ (see Materials and Methods). Whether the ICCls-ICX connection exhibits more widely spaced contacts, and whether those ICDs are subject to prism-induced remodeling as documented here for shorter-range contacts, remains an open question.

Nonlinear dendritic summation is not a universal finding (Cash and Yuste, 1999; Walter and Khodakhah, 2006) and could be cell type specific. There is no incisive whole-cell recording data for the CaMKII+ ICX neurons we studied. Intracellular sharp electrode recordings from space-specific neurons in ICX, a population that overlaps significantly with the CaMKII+ population, have established their capacity to perform nonlinear operations including the multiplication of inputs (Pena and Konishi, 2001). Whether individual CaMKII+ dendrites act as independent computational subunits capable of nonlinear integration is unknown.

\section{Balanced creation and elimination of contacts during learning}

The adaptive zone in normal juveniles contains both closely spaced $(<20 \mu \mathrm{m})$ and more dispersed $(>20 \mu \mathrm{m})$ contacts, whereas in prism-adapted owls, only closely spaced contacts were observed (Fig. $8 \mathrm{~A}$ ). We consider three possible explanations: (1) formation of new contacts during prism-induced learning, (2) contact elimination, or (3) both. In the first model, the creation of new contacts intercalated between widely spaced ones would eliminate the occurrence of long ICDs, as was observed in the data. However, this process 
would also result an increase in the number of contacts per branch of dendrite, which was not observed (Fig. $7 A, B$ ). In the second model, widely spaced contacts are eliminated. This implies either a reduction in the number of contacts per branch, or reduction in the proportion of dendrites receiving contacts. Neither was observed (Fig. 7). In the third model, the creation of new, closely spaced contacts is balanced in number by elimination of widely spaced ones. This predicts no change in the number of contacts per branch or proportion of dendrites receiving contacts, which is consistent with the data (Fig. 7). A similar line of reasoning applies to the declustering of contacts that was observed in the normal zone. Thus, the changes in contact clustering must have resulted from both contact formation and contact elimination (Fig. 13).

How are the patterns of clustering independently sculpted in each zone? Neurons in ICX receive an instructive signal that encodes the adaptive value of presynaptic stimulus with postsynaptic response (Knudsen, 1994). For example, on the R side of the brain of prism owls, only those contacts located on the rostral flank of the ICCls arbor innervate CaMKII+ neurons that project to the correct (behaviorally adaptive) map locations within OT. The instructive signal received by these CaMKII + neurons is positive (i.e., it need promote strengthening of those auditory inputs). Conversely, neurons in the normal or maladaptive zone require no strengthening, and receive a neutral or negative signal. Thus, the simplest possibility is that the instructive signal acts to stabilize coactive clusters of ICClsCaMKII + contacts, and to destabilize desynchronous clusters.

\section{Complementary mechanisms that fine-tune circuit function}

Synapse clustering represents a novel mechanism for optimizing circuit function during learning, but is not the only factor that determines the shape of the auditory tuning curves. The bulk spatial density of ICCls axons and boutons distributed across the ICCls arbor is highest where auditory-driven responses are strongest. Yet this correlation is imperfect, because the density in the normal zone of prism-adapted owls is also high despite the expression of weak responses (DeBello et al., 2001). The functional weakening of normal responses, which correlates well with the prism-induced declustering of contacts documented here, can also be accounted for by topographic changes in the strength of GABAergic inhibition (Zheng and Knudsen, 1999). Finally, long durations of prism experience lead to an AMPAfication of adaptive synapses (Feldman and Knudsen, 1998b), strong evidence of synapse strengthening via long-term potentiation. This effect, however, cannot explain the initial appearance of adaptive responses, because a related study found these are heavily depen- dent on NMDA receptors (Feldman and Knudsen, 1998a), expected to deliver weaker drive.

\section{Future experiments}

These results raise several questions that can be addressed using the framework developed here: (1) Does learning in the adult brain involve microanatomical reorganization? When prisms are removed from adult owls, auditory tuning reverts to its native configuration as ICX neurons reexpress strong normal responses and suppress adaptive ones. Our model predicts an increase in clustering in the normal zone and a decrease in the adaptive zone. A related line of adult plasticity experiments asks whether clustering is adjusted during enhancement paradigms, incremental training (Linkenhoker and Knudsen, 2002) or active versus passive feeding (Bergan et al., 2005). (2) What are the dynamics that lead to more contact clustering? One possibility is that new contacts initially form at random locations along the dendrite and are then subject to Hebbian-like selection: clustered contacts are stabilized, whereas isolated contacts are eliminated. A second possibility is that the instructive signal directs the spatially precise cre- 


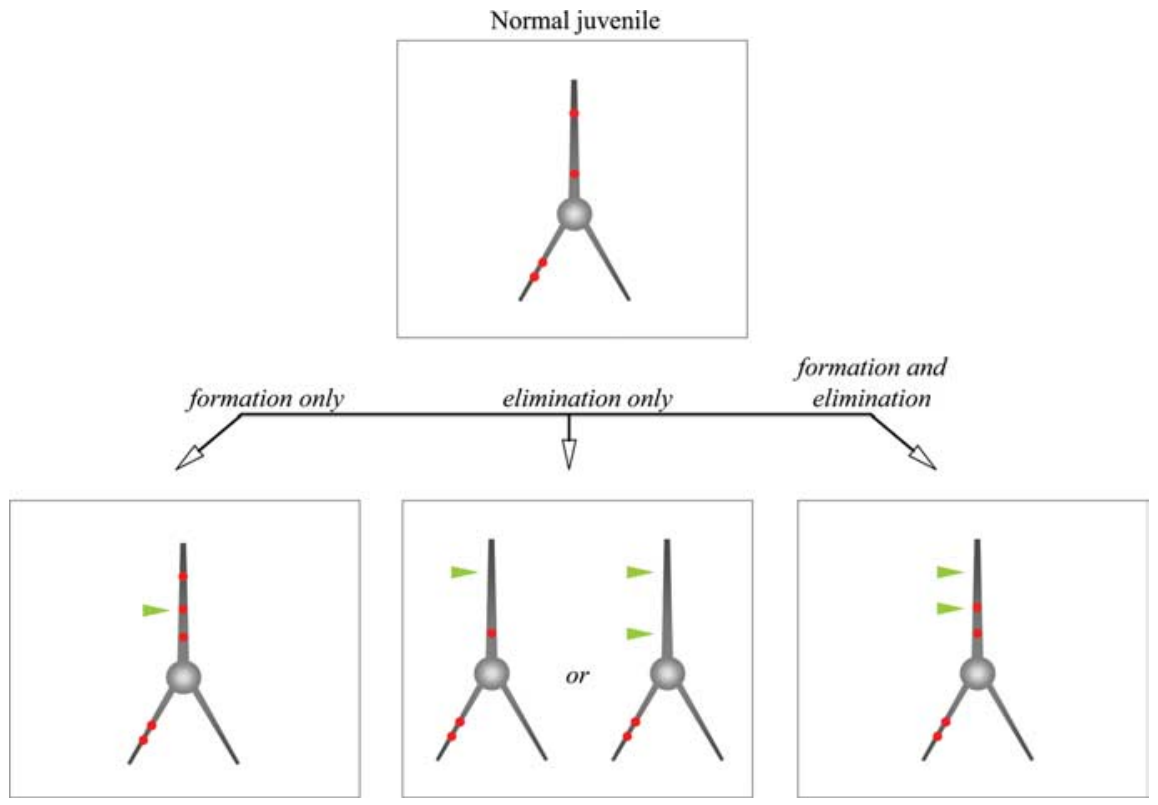

Figure 13. Schematic diagram of results. Diagram of prism-induced changes in contact clustering within the adaptive zone. For simplicity, contacts are shown onto a single neuron. Dendrites are depicted as gray cylinders, and axodendritic contacts are depicted as red dots. The right space zone in normal juveniles (top) exhibits both short-range $(<20 \mu \mathrm{m}$ ) and longer-range ( $>20 \mu \mathrm{m}$ ) contacts. Three models are presented to account for the prism-induced disappearance of widely spaced contacts in the corresponding adaptive zone of prism owls: one dependent solely on the formation of new contacts (bottom left), one dependent solely on contact elimination (bottom middle), and one dependent on both formation and elimination (bottom right). The locations of contact changes are delineated with green arrows. Only the latter model is consistent with the data.

ation of new contacts only within the vicinity of preexisting ones. This question can be addressed by analysis of owls in early stages of prism adaptation. (3) Does the capacity for microanatomical reorganization extend to other circuit elements in ICX? The CaMKII+ neurons studied here are exclusively excitatory. Yet the integration of excitatory and inhibitory networks within ICX plays a major role in shaping auditory responses (Fujita and Konishi, 1991; Zheng and Knudsen, 1999, 2001). A connectionist model has been proposed account for learning-related changes in inhibitory network function (DeBello and Knudsen, 2001). This model can be tested by applying the methods described here to the dendritic fields of inhibitory neurons, visualized using a combination of lentivirusmediated GFP (green fluorescent protein) expression and retrospective immunohistochemistry.

\section{References}

Augustine GJ, Santamaria F, Tanaka K (2003) Local calcium signaling in neurons. Neuron 40:331-346.

Bergan JF, Ro P, Ro D, Knudsen EI (2005) Hunting increases adaptive auditory map plasticity in adult barn owls. J Neurosci 25:9816-9820.

Brainard MS, Knudsen EI (1993) Experience-dependent plasticity in the inferior colliculus: a site for visual calibration of the neural representation of auditory space in the barn owl. J Neurosci 13:4589-4608.

Cash S, Yuste R (1999) Linear summation of excitatory inputs by CA1 pyramidal neurons. Neuron 22:383-394.

Chklovskii DB, Mel BW, Svoboda K (2004) Cortical rewiring and information storage. Nature 431:782-788.

DeBello WM, Knudsen EI (2001) Adaptive plasticity of the auditory space map. In: Toward a theory of neuroplasticity (Shaw CA, McEachern JC, eds), pp 13-30. Philadelphia: Psychology.

DeBello WM, Feldman DE, Knudsen EI (2001) Adaptive axonal remodeling in the midbrain auditory space map. J Neurosci 21:3161-3174.
De Paola V, Holtmaat A, Knott G, Song S, Wilbrecht L, Caroni P, Svoboda K (2006) Cell typespecific structural plasticity of axonal branches and boutons in the adult neocortex. Neuron 49:861-875.

Feldman DE, Knudsen EI (1997) An anatomical basis for visual calibration of the auditory space map in the barn owl's midbrain. J Neurosci 17:6820-6837.

Feldman DE, Knudsen EI (1998a) Pharmacological specialization of learned auditory responses in the inferior colliculus of the barn owl. J Neurosci 18:3073-3087.

Feldman DE, Knudsen EI (1998b) Experiencedependent plasticity and the maturation of glutamatergic synapses. Neuron 20:1067-1071.

Fujita I, Konishi M (1991) The role of GABAergic inhibition in processing of interaural time difference in the owl's auditory system. J Neurosci 11:722-739.

Gasparini S, Magee JC (2006) State-dependent dendritic computation in hippocampal CA1 pyramidal neurons. J Neurosci 26:2088-2100.

Gold JI, Knudsen EI (2000) A site of auditory experience-dependent plasticity in the neural representation of auditory space in the barn owl's inferior colliculus. J Neurosci 20:3469-3486.

Holtmaat A, Wilbrecht L, Knott GW, Welker E, Svoboda K (2006) Experience-dependent and cell-type-specific spine growth in the neocortex. Nature 441:979-983.

Hua JY, Smith SJ (2004) Neural activity and the dynamics of central nervous system development. Nat Neurosci 7:327-332.

Knudsen EI (1983) Subdivisions of the inferior colliculus in the barn owl (Tyto alba). J Comp Neurol 218:174-186.

Knudsen EI (1994) Supervised learning in the brain. J Neurosci 14:3985-3997.

Knudsen EI (2002) Instructed learning in the auditory localization pathway of the barn owl. Nature 417:322-328.

Knudsen EI, Knudsen PF (1985) Vision guides the adjustment of auditory localization in young barn owls. Science 230:545-548.

Konishi M (2003) Coding of auditory space. Annu Rev Neurosci 26:31-55.

Le Be JV, Markram H (2006) Spontaneous and evoked synaptic rewiring in the neonatal neocortex. Proc Natl Acad Sci U S A 103:13214-13219.

Lee WC, Huang H, Feng G, Sanes JR, Brown EN, So PT, Nedivi E (2006) Dynamic remodeling of dendritic arbors in GABAergic interneurons of adult visual cortex. PLoS Biol 4:e29.

Linkenhoker BA, Knudsen EI (2002) Incremental training increases the plasticity of the auditory space map in adult barn owls. Nature 419:293-296.

Linkenhoker BA, von der Ohe CG, Knudsen EI (2005) Anatomical traces of juvenile learning in the auditory system of adult barn owls. Nat Neurosci 8:93-98.

Lu J, Helton TD, Blanpied TA, Racz B, Newpher TM, Weinberg RJ, Ehlers MD (2007) Postsynaptic positioning of endocytic zones and AMPA receptor cycling by physical coupling of dynamin-3 to Homer. Neuron $55: 874-889$.

Nagerl UV, Eberhorn N, Cambridge SB, Bonhoeffer T (2004) Bidirectional activity-dependent morphological plasticity in hippocampal neurons. Neuron 44:759-767.

Pena JL, Konishi M (2001) Auditory spatial receptive fields created by multiplication. Science 292:249-252.

Poirazi P, Mel BW (2001) Impact of active dendrites and structural plasticity on the memory capacity of neural tissue. Neuron 29:779-796.

Poirazi P, Brannon T, Mel BW (2003) Arithmetic of subthreshold synaptic summation in a model CA1 pyramidal cell. Neuron 37:977-987. 
Polsky A, Mel BW, Schiller J (2004) Computational subunits in thin dendrites of pyramidal cells. Nat Neurosci 7:621-627.

Rodriguez-Contreras A, Liu XB, DeBello WM (2005) Axodendritic contacts onto calcium/calmodulin-dependent protein kinase type IIexpressing neurons in the barn owl auditory space map. J Neurosci 25:5611-5622.

Sala C, Piech V, Wilson NR, Passafaro M, Liu G, Sheng M (2001) Regulation of dendritic spine morphology and synaptic function by Shank and Homer. Neuron 31:115-130.

Schneider Gasser EM, Straub CJ, Panzanelli P, Weinmann O, SassoePognetto M, Fritschy JM (2006) Immunofluorescence in brain sections: simultaneous detection of presynaptic and postsynaptic proteins in identified neurons. Nat Protoc 1:1887-1897.
Stettler DD, Yamahachi H, Li W, Denk W, Gilbert CD (2006) Axons and synaptic boutons are highly dynamic in adult visual cortex. Neuron 49:877-887.

Swofford JA, DeBello WM (2007) Transcriptome changes associated with instructed learning in the barn owl auditory localization pathway. Dev Neurobiol 67:1457-1477.

Walter JT, Khodakhah K (2006) The linear computational algorithm of cerebellar Purkinje cells. J Neurosci 26:12861-12872.

Zheng W, Knudsen EI (1999) Functional selection of adaptive auditory space map by GABAA-mediated inhibition. Science 284:962-965.

Zheng W, Knudsen EI (2001) GABAergic inhibition antagonizes adaptive adjustment of the owl's auditory space map during the initial phase of plasticity. J Neurosci 21:4356-4365. 